\title{
Cantrell Syndrome. Case Report of an Adult
}

\author{
João Luiz A lencar Araripe Falcão, Sandra N ívea dos Reis Saraiva Falcão, W anda Cristina Sawicki, \\ Aroldo W alter Liberatori Fo, A ntonio Carlos Lopes
}

São Paulo, SP - Brazil

\begin{abstract}
Cantrell syndrome is characterized by defects that involve the diaphragm, abdominal wall, pericardium, heart, and lower region of the sternum. It is a rare entity, usually diagnosed at birth and accompanied by high mortality due to the complexity and gravity of the anomalies. In this report, we present a 32-year-old male patient, who was diagnosed in infancy but who reached adult age asymptomatic.
\end{abstract}

Cantrell syndrome is a rare entity, characterized by malformation of the lower portion of the sternum, ventral region of the diaphragm, and mid- abdominal section, associated with cardiac anomalies ${ }^{1}$. Previously described as ectopia cordis associated with other anomalies, in 1958 it was presented by Cantrell et $\mathrm{al}^{2}$ as a unique syndrome, resulting from lack of development of the anterior/frontal mesoderm during embryonic life.

The syndrome is usually detected at birth; however, it is still a surgical challenge due to the complexity of the congenital malformations, which determine a low life expectancy ${ }^{1,3}$.

We report a rare case of this syndrome diagnosed in adult age in, until recently, an asymptomatic patient, with tetralogy of Fallot, anomalous venous drainage, ventricular diverticula, and coronary arteries originating from only one trunk.

\section{Case Report}

A male patient, now 32 years old, the son of nonconsanguineous parents, was admitted to the ward for clinical investigation of congenital cardiopathy discovered during preoperative examinations for surgical correction of omphalocele during the first year of life. At that time, surgical correction of the congenital cardiac disease was proposed, but as the patient was asymptomatic and had normal psychomotor development, treatment was always postponed by the family.

Universidade Federal de São Paulo - Escola Paulista de Medicina

Mailing address: João Luiz Alencar Araripe Falcão - Rua Caribe, 51 - 04562-060 - São Paulo, SP - Brazil
The patient remained asymptomatic until recently, when he began to experience progressive edema in the lower limbs and dyspnea during stress, requiring hospitalization for clinical compensation. Later, the patient evolved with repeated episodes of tachycardia without hemodynamic repercussion. Twenty-four hour Holter monitoring demonstrated sustained atrial flutter with variable atrioventricular conduction. The electrophysiology study showed an arrhythmogenic focus, where treatment by ablation was unsuccessful, followed by ectopic atrial rhythm with low ventricular response.

The patient was admitted in good general condition, with jugular stasis ++ at $45^{\circ}$, hemangioma at the left hemifacies, a xiphoid appendix noticeable on palpation, a flaw in the coaptation of the left lower costal arches, and with evident epigastric pulsation. The precordium examination showed systolic rise and palpable, roaring cardiac propedeutic ++ at the $3^{\text {rd }}$ right intercostal space and rhythmic, normophonetic heart sounds, not tachycardiac, with hyperphonetic $\mathrm{B}_{2}$ in the area of pulmonary auscultation and a rough holosystolic murmur +++ in the $3^{\text {rd }}$ right intercostal space irradiating to the entire frontal thoracic region. The abdomen examination showed the liver palpable at $2 \mathrm{~cm}$ from the right costal edge, hardened, with a blunt edge, and smooth and painful superficies.

The thorax X-ray showed dextrorotatory mesocardia, images suggesting an increase in the right cardiac chambers, arching of the pulmonary arterial trunk and prominent pulmonary hila. Transthoracic and transesophageal echocardiography revealed a left ventricle with intact contractile function, great perimembranous interventricular communication, with a left - right shunt on Doppler, infundibular pulmonary stenosis, right ventricular hypertrophy, a significant increase in the right atrium, tricuspid failure, and an aorta significantly mounting the interventricular septum. The angiographic study furthermore showed the presence of anomalous systemic venous drainage (superior vena cava draining to left atrium), diverticula in both ventricles and coronary arteries originating from only one trunk (fig. 1). Thoracic-abdominal nuclear magnetic resonance imaging showed the absence of the frontal and central portion of the diaphragm and the angiographic data above mentionned (fig. 2-3). With these results, the diagnosis of Cantrell syndrome, in an adult patient, was established. 


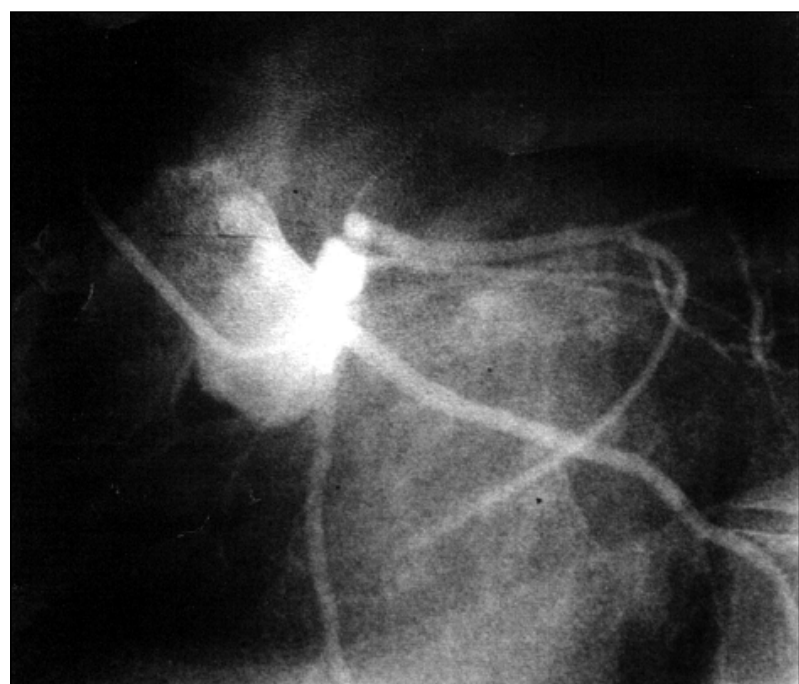

Fig. 1 - Cinecoronariography in OAE showing coronary arteries originating from one trunk.

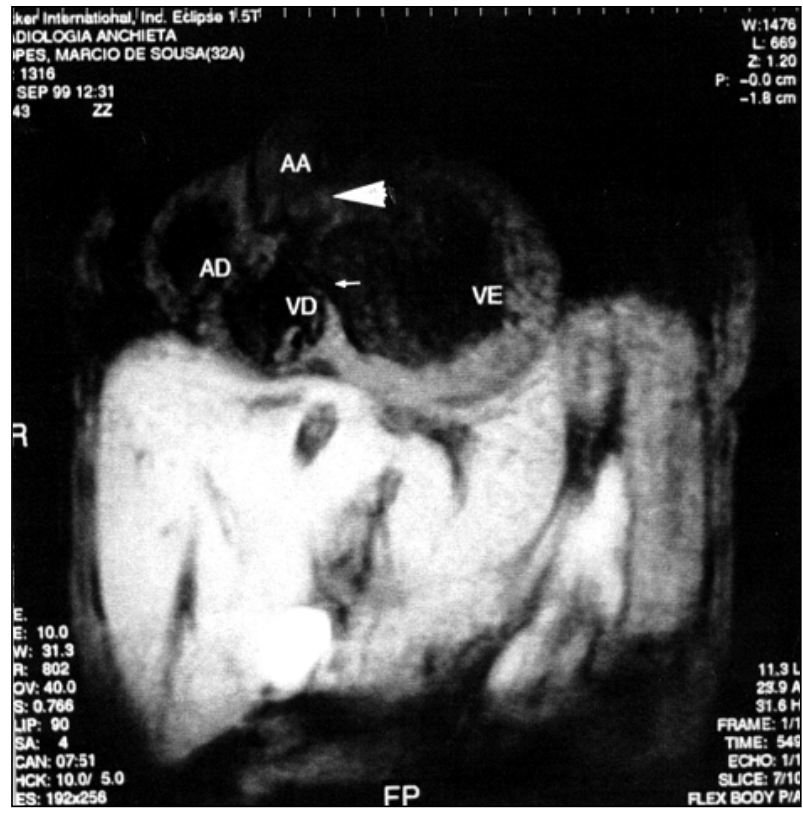

Fig. 2 - Cardiac nuclear magnetic resonance image (coronal section) showing interventricular communication (arrow) and the aorta mounting the interventricular septum (head of arrow).

\section{Discussion}

In 1958, Cantrell, Haller, and Ravitch ${ }^{3}$ described the syndrome of Cantrell, which was characterized by five anomalies: malformation of the lower portion of the sternum, supra-umbilical abdominal wall, ventral region of the diaphragm, a diaphragmatic pericardium, and cardiac anomalies. Vazquez-Jimenez et $\mathrm{al}^{4}$, in an ample review of the literature, reported 153 cases of the syndrome in its complete or incomplete form.

Its pathogeny is still unknown. In spite of the apparent lack of genetic input (participation) a high prevalence of trisomy of chromosome 13 or 18 in patients with conge-

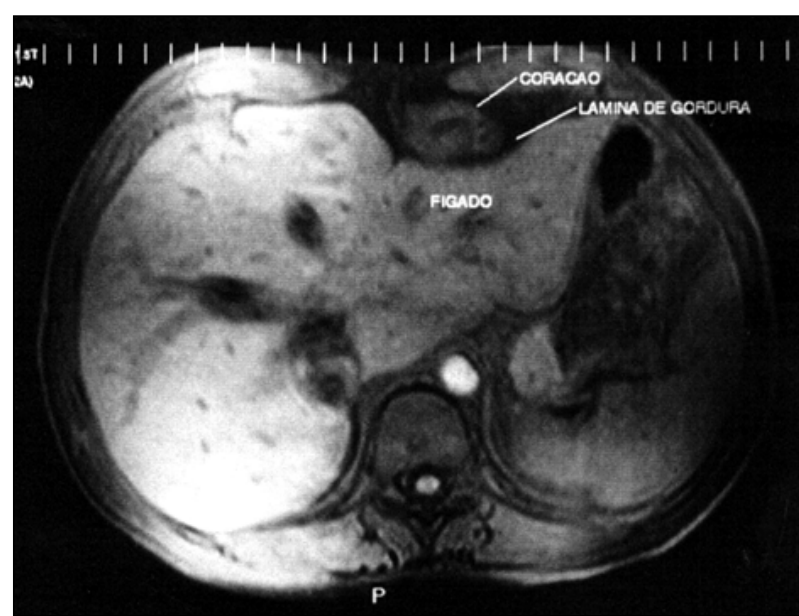

Fig. 3 - Thoracic - abdominal nuclear magnetic resonance image (axial section) showing the lack of a frontal region of the diaphragm and of pericardium.

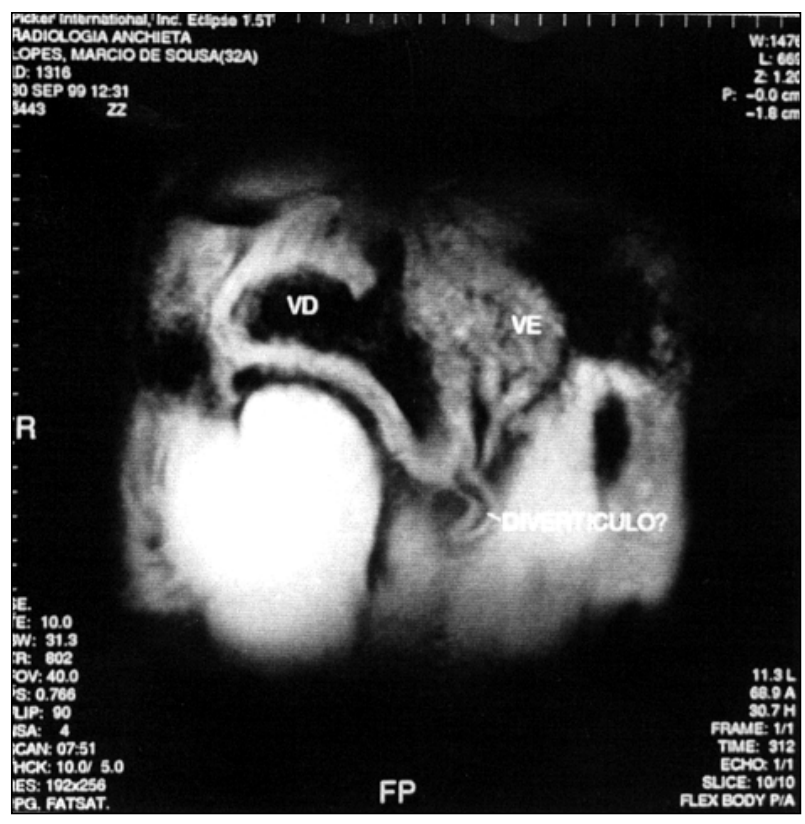

Fig. 4 - Cardiac nuclear magnetic resonance image (coronal section) showing right ventricular diverticula.

nital cardiopathy and omphalocele and as, already described, cases of the syndrome involving twins ${ }^{1,5}$ was observed.

Cantrell established that the syndrome may be due to the lack of differentiation of intraembryonic mesoderm, between the splanchnic/viscera and the wall, during the $14^{\text {th }}$ and $18^{\text {th }}$ days of embryonic life ${ }^{3}$. Barrow and Willis ${ }^{2}$ induced, with the use of beta-aminopropionitrile in an experimental model, ectopia cordis and gastroschisis. Defects in differentiation of the mesoderm may cause failure in cardiac levorotation, cardiac malformation, omphalocele, and defects in coaptation of the sternal band and ribs ${ }^{1,4,5}$.

The syndrome of Cantrell mainly prevails in males (57.5\%), with an estimated incidence of 1:65,000 live births. In relation to the anomalies found in the syndrome, abdomi- 
nal wall malformation occurs in $74.5 \%$; extruding omphalocele of the lower sternum, in 59.4\%; of the diaphragm in $56.8 \%$; mainly in the ventral region, and of the pericardium in $41.8 \%$, and its absence determines visible and palpable epigastric pulsation. In relation to cardiac anomalies, they occur in $83 \%$ of cases; interventricular communication is the most prevalent. Interventricular communication, ventricular diverticula, stenosis or pulmonary artery atresia, dextrocardia, anomalous left superior vena cava, anomalous pulmonary venous return, truncus arteriosus, ventricular aneurysm, and tetralogy of Fallot have a smaller incidence ${ }^{1,4,5}$.

Dyspnea and central cyanosis are frequently found ${ }^{6}$. A chest X -ray may show dextrocardia, other thoracic anomalies, or both. Echocardiography, nuclear magnetic resonance imaging, and hemodynamic studies show with precision the associated cardiac defects ${ }^{3,5}$.

The patient in this case study had the complete form of pentalogy of Cantrell: omphalocele, mesocardia with dextrorotation, anomalous venous drainage of the left superior vena cava, coronary arteries originating from only one trunk, tetralogy of Fallot, ventricular diverticula, malformation of the lower third of the sternum, malformation of the diaphragmatic pericardium, and malformation in the anterior/ frontal and central wall of the diaphragm.

Surgical treatment makes the necessary correction of cardiac, abdominal, and thoracic defects. However, controversies exist in the literature regarding the best surgical tactic ${ }^{4}$. Usually, the precocious correction of the defects is chosen, taking into consideration the gravity of the malformation ${ }^{5}$. Correction of cardiac anomalies must be prioritized in patients with low cardiac output at the preoperative examination ${ }^{6}$. However, in patients where those malformations are not very expressive, surgical correction should focus on the omphalocele and diaphragmatic alterations because treatment when carried out first in infancy is technically easier and avoids the risk of external cardiac compression and pulmonary hypoplasia ${ }^{4,6}$. The ideal period for surgical correction of omphalocele depends on its size and the material contained ${ }^{5}$. The whole and well-epithelialized omphalocele do not require emergency correction; however, rupture or imminent risk of rupture make surgery imperative, due to the possibility of the onset of mediastinitis, peritonitis, or both ${ }^{5,6}$.

Precocious resection of ventricular diverticula must always be considered due to the high risk of traumatic or spontaneous rupture and; sudden death due to tachyarrhythmias $(6-20 \%)^{4}$.

The cardiac defects present in Cantrell syndrome are the determinants of the high rate of morbidity and mortality and may constitute limiting factors in surgical corrections of other associated defects ${ }^{4-6}$. In summary, the syndrome of Cantrell is an extremely rare entity, where patients usually do not reach adulthood, principally due to the complexity of cardiac anomalies. In this case report, the patient reached adulthood, in spite of having the complete form of the syndrome, and remained asymptomatic until recently when, at this hospitalization, surgical treatment of cardiopathy was proposed.

\section{References}

1. Baker ME, Rosenberg ER, Trofatter KF, Imber MJ, Bowie JD. The utero findings in twins pentalogy of Cantrell. J Ultrasound Med. 1984; 3: 525-7.

2. Barrow MV, Willis LS. Ectopia cordis (ectocardia) and gastroschisis induced in rats by maternal administration of lathyrogen, beta-aminopropionitrile (BAPN). Am Heart J 1972; 83: 518-26.

3. Cantrell JR, Haller JA, Ravitch MM. A syndrome of congenital defects involving the abdominal wall, sternum, diaphragm, pericardium and heart. Surg Gynecol Obstet 1958; 107: 602-4.
4. Vazquez-Jimenez JF, Muehler EG, Daebritz S, et al. Cantrell's Syndrome:A challenge to the surgeon. Ann Thorac Surg 1998; 65: 117885 .

5. Ghidini A, Sirtori M, Romero R, Hobbins JC. Prenatal diagnosis of pentalogy of Cantrell. J Ultrasound Med 1988; 7: 567-72.

6. Hornberger LK, Colan SD, Lock JE, Wessel DL, Mayer JE Jr. Outcome of patients with ectopia cordis and significant intracardiac defects. Circulation 1996; 94(suppl II): II-32-7. 